\title{
Der Ritalinkonsum steigt weiter an
}

\section{Hans Rudolf Stricker}

Facharzt für Allgemeinmedizin kognitive Verhaltenstherapie, Praxis für Erwachsene mit ADHS

Korrespondenz:

Dr. med. Hans Rudolf Stricker

Mattengasse 52

CH-8005 Zürich

Tel. 0442717333

hr.stricker[at]hin.ch
Die Statistiken des Heilmittelinstituts Swissmedic und des Krankenversicherers Helsana belegen, dass in der Schweiz immer mehr Methylphenidat [1] verabreicht wird. Die ärztlich verordneten Mengen des Wirkstoffs nehmen seit Mitte der Neunzigerjahre kontinuierlich zu, seit 2007 jedes Jahr um durchschnittlich 15\%.

Auch für 2011 sind die Liefermengen an den Detailhandel innerhalb eines Jahres um $49 \mathrm{~kg}$ auf $343 \mathrm{~kg}$ angestiegen, wobei laut Swissmedic der Anstieg grösstenteils auf eine Änderung in der Datenerhebung (präziser) zurückzuführen sei. Dass jedoch Dexmethylphenidat (Focalin $\mathrm{XR}^{\circledast}$ ) doppelt so stark wie Ritalin ${ }^{\circledast}$, das heisst z.B. 5 mg Focalin $\mathrm{XR}^{\circledast}$ etwa gleich stark wie 10 mg Ritalin ${ }^{\circledast}$ wirkt, wird bei dieser Mengenaddition für die verschiedenen Präparate nicht berücksichtigt. Diese Darstellung kaschiert somit bei zunehmender Focalinverschreibung den steigenden Stimulanziengebrauch perfekt. Somit dürfte der reale Anstieg des Verbrauchs für das Jahr 2011 (Äquipotenz mitberücksichtigt) in ähnlichem Rahmen wie in den vorangegangenen Jahren weitergehen.

Diese Entwicklung wird von vielen Schweizern mit Sorge betrachtet. Was müssen wir tun, um nicht ins «Fahrwasser» der liberalen Ritalinverschreibung zu geraten, so wie es in den letzten zwei Jahrzehnten

\section{Methylphenidat in der Schweiz: Liefermengen 1996-2010}

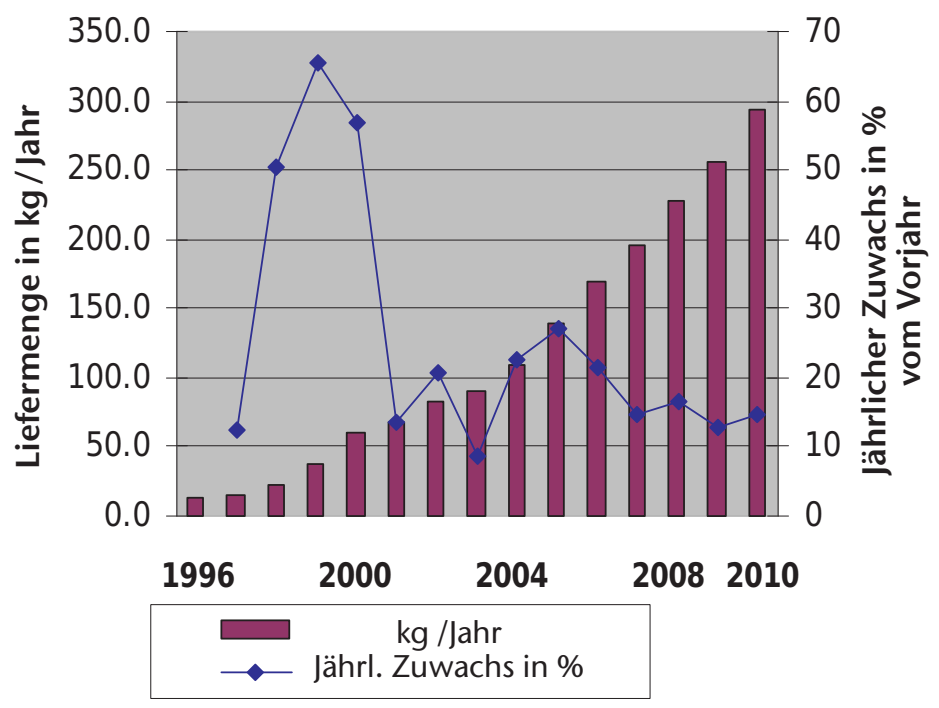

\section{La consommation de ritaline continue d'augmenter}

Le méthylphénidate [1] (par ex. Ritaline ${ }^{\circledast}$ ) est de plus en plus employé dans beaucoup d'affections. Hormis le trouble du déficit de l'attention/hyperactivité (TDA/H), il est utilisé comme stimulant du cerveau et, plus rarement, pour de nombreux autres troubles physiques et psychiques. Pour contenir cette augmentation, des mesures appropriées sont nécessaires. Premièrement, nous avons besoin de davantage de spécialistes du TDA/H qualifiés qui posent des diagnostics clairs et travaillent avec des plans thérapeutiques personnalisés. Deuxièmement, les sociétés de discipline médicale et les associations professionnelles sont appelées à rédiger de nouvelles directives ciblées dans ce domaine, contenant notamment des dispositions en matière de formation continue, afin de garantir une prise en charge de qualité aux patients atteints de TDA/H. Enfin, il est important que chaque médecin connaisse ses limites et qu'il ne diagnostique et ne traite sous sa propre responsabilité que les affections pour lesquelles il a été formé et dans lesquelles il a acquis de l'expérience sous la supervision de spécialistes.

in den USA geschehen ist? Auch Politiker beschäftigen sich damit und haben in den eidgenössischen und jüngst in kantonalen Parlamenten Studien zur Ritalinverschreibung beantragt.

\section{Vielfacher Nutzen bei ADHS}

Tatsächlich hat sich die Verabreichung von Methylphenidat bei eindeutig diagnostizierter Aufmerksamkeitsdefizit-/Hyperaktivitätsstörung (ADHS) [2] als eine wirksame Therapie erwiesen, insbesondere dann, wenn ein ADHS-Spezialist die Krankheit sowie auch Nebenerkrankungen (Komorbiditäten) sorgfältig abklärt und die Medikation überwacht (Evidenz- 
stufe Ia, Empfehlungsgrad A). Der persönliche, gesellschaftliche und ökonomische Nutzen bei fachgerechter Methylphenidatbehandlung ist gross, ganz besonders bei schwereren ADHS-Formen und in Kombination mit Coaching oder Psychotherapie.

\section{Mehr gute Abklärungs- und Behandlungsstellen sind dringend nötig.}

Die Nebenwirkungen des Wirkstoffes sind bei einer qualifizierten Stimulanzienverschreibung und bei Vorliegen einer klaren ADHS-Diagnose gering: Den seltenen und gefährlichen Folgen wie Abhängigkeit, Veränderung der Persönlichkeit, chronische Hirnschädigung oder plötzlicher Herztod kann bei genauem Erfragen der Nebenwirkungen und exakter Erhebung der Anamnese bezüglich Risikofaktoren präventiv gut vorgebeugt werden.

\section{Methylphenidat wird vielfältig angewendet}

Nach Swissmedic besteht noch bei der Narkolepsie eine Indikation für «Ritalin», wobei hier fast nur das «modernere» Modafinil eingesetzt wird.

Bei vielen weiteren Diagnosen der Psychiatrie und der Körpermedizin wird Methylphenidat «Off-label» selten eingesetzt oder ausprobiert. So z. B. bei Patienten mit auffallenden kognitiven Symptomen, Störun- gen der exekutiven Funktionen (z. B. planen, Impulse hemmen), Gedächtnisstörungen, innerer Unruhe, äusserer Hyperaktivität, Impulsivität, Affektausbrüchen und emotionaler Instabilität, aber auch bei körperlicher Erschöpfung und Müdigkeit. Zwar können viele dieser Einzelsymptome auch bei ADHS vorkommen, um jedoch die Diagnose ADHS zu erfüllen, müssten mehrere dieser Symptome nebeneinander, so wie sie im DSM-IV oder in ICD-10 beschrieben sind, vorhanden und zudem die Zusatzkriterien erfüllt sein.

Eine fragliche ADHS-Diagnose, meist als «Verdacht auf ADHS» formuliert, wird oft gerade deshalb gestellt, um einen «Ritalinversuch» zu legitimieren. Bei genauerem Hinschauen eines Spezialisten, d.h. Abklären von Differentialdiagnosen, würden sich viele dieser Verdachtsdiagnosen als Fehldiagnosen herausstellen.

Der gelegentliche Versuch, Suchtkranke vom Kokaintyp mit Methylphenidat zu substituieren, führt fast nie aus der Sucht und sollte nicht angewendet werden.

Im Sinne eines körperlichen Dopings werden Stimulanzien nicht nur im Leistungssport, sondern auch zum besseren Durchhalten von langen Partynächten eingesetzt.

\section{Hirndoping}

Immer öfter wird bei Managern mit hohem Leistungsstress und bei Mittelschülern und Studenten, die vor Prüfungen oder unter starkem Leistungsdruck

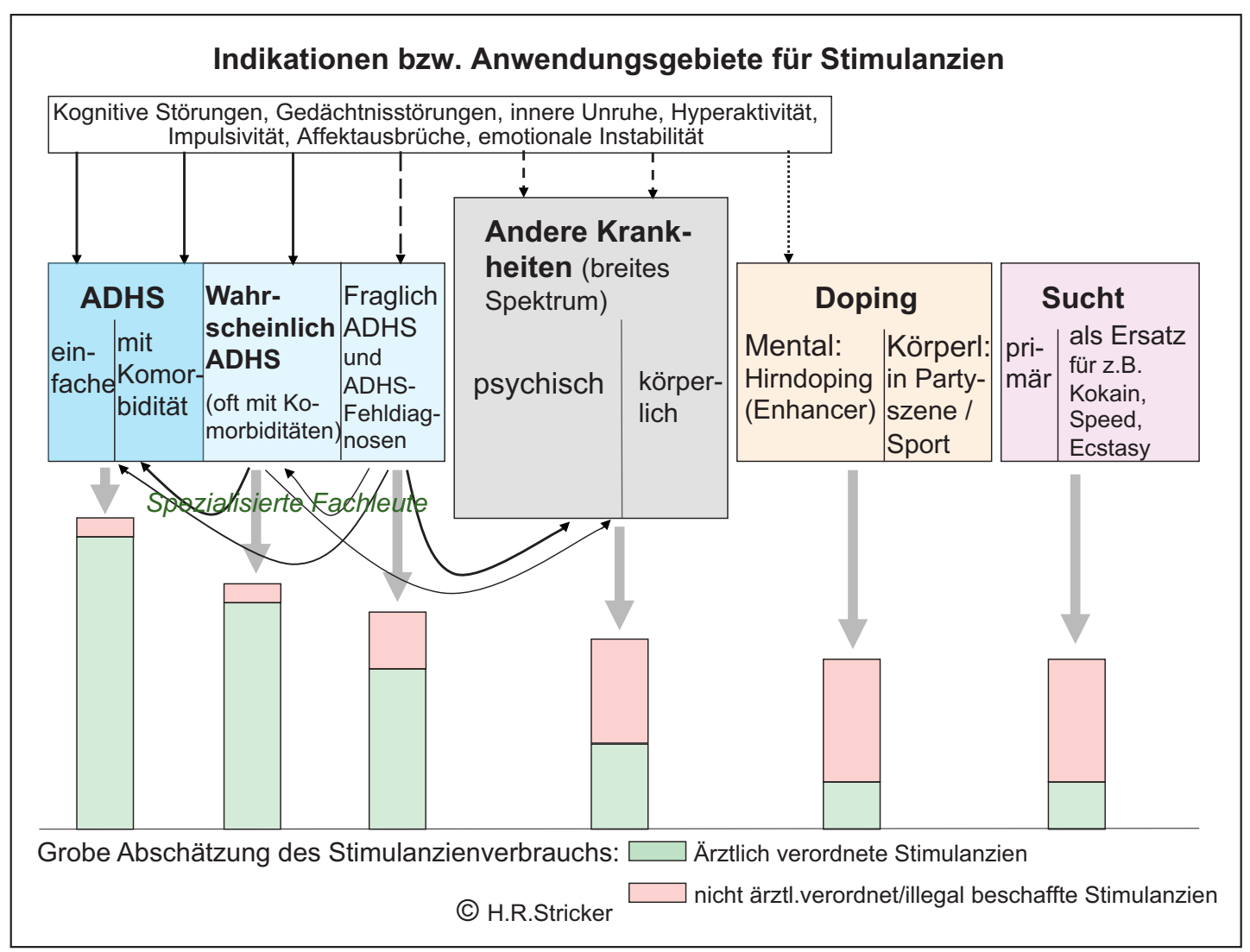


stehen, Methylphenidat als Hirndoping (Enhancer) eingesetzt. Nach wissenschaftlichen Untersuchungen wird die Aufmerksamkeit bei nicht von ADHS Betroffenen nicht verbessert. Einen wachmachenden Effekt hat Methylphenidat nachweislich bei Übermüdung. Zudem bewirkt es ein zuversichtliches Herangehen an schwierige Aufgaben und hat also aufgrund der positiven Wirksamkeitserwartung (Plazeboeffekt) einen günstigen Einfluss auf die Leistung. Genügend Schlaf, entspannende Pausen und eine abwechslungsreiche Freizeitgestaltung sind aber eine weit bessere «Medizin» als die Einnahme von stimulierenden Pillen. aufklärende und stützende Gespräche oder ein Coaching in ihren Problemlösungsstrategien im Arbeitsund Familienumfeld.

Um dem beginnenden «Wildwuchs» von oberflächlich gestellten ADHS-Diagnosen und der unkritischen, zu grosszügigen Verschreibung von Stimulanzien entgegenzuwirken, sind Qualifizierungskriterien für Ärzte gefordert. Diese könnten durch die involvierten Facharztgruppen und Fachgesellschaften ausgearbeitet werden. Kinder-/Jugend- und Erwachsenenpsychiater sowie Pädiater, d.h. die Fachärzte der drei Kern-Fachgesellschaften und wenige andere Fachärzte (z. B. Allgemeine Innere Medizin),

\section{Ärzte, die ADHS-Patienten behandeln, sollen ADHS-spezifische Fortbildungen in Qualitätszirkeln, Supervisionen, aber auch Kongresse und Kurse besuchen.}

Zudem besteht bei häufigem Konsum von Stimulanzien bei einem grossen Teil von nicht an einer ADHS Erkrankten die Gefahr, in eine psychische Abhängigkeit abzugleiten. Bemerkenswert ist, dass Stimulanzien, die zu Dopingzwecken, bei Sucht- und gelegentlich anderen psychiatrischen Erkrankungen eingesetzt werden, oft übers Internet bestellt werden, bei Dealern gekauft oder von Personen bezogen werden, die diese Substanzen ursprünglich von einem Arzt verschrieben bekommen haben.

\section{Mehr auf ADHS spezialisierte Ärzte sind nötig}

Die ADHS ist eine häufige Krankheit, die ca. bei 4-8\% der Kinder auftritt und sich nur in knapp der Hälfte der Fälle im Laufe der Pubertät auswächst, was bedeutet, dass 2-5\% der Erwachsenen ebenfalls von ADHS betroffen sind. Mehr gute Abklärungs- und Behandlungsstellen sind deshalb dringend nötig.

Die ADHS-Diagnostik ist durch die Überlagerung der ADHS-Symptomatik von anderen psychiatrischen Krankheiten (60-70\% haben komorbide Störungen) schwierig, ebenso wie die Abgrenzung, ob es sich um eine Komorbidität handelt oder eben doch eine differentialdiagnostisch erwogene Krankheit der ADHSDiagnose vorzuziehen ist. Dazu wie auch für die umfassende multimodale Therapie braucht es auf ADHS spezialisierte Ärzte und Psychologen. Eine Abgabe von Stimulanzien bei falscher Indikation an nicht von ADHS Betroffene wirkt selten positiv und birgt zudem die Gefahr einer Suchtentwicklung. Die Entscheidung, ob Stimulanzien oder andere Medikamente indiziert sind, die anspruchsvolle, feine Eintitrierung der Stimulanziendosierung, das begleitende Coaching und evtl. Psychotherapie bedürfen viel Wissen und Erfahrung auf diesem Gebiet. Menschen mit einer leichten Form von ADHS brauchen meist keine Medikamente, profitieren aber durch gezielte, müssten nach meiner Ansicht, wenn sie ADHS-Patienten behandeln, ADHS-spezifische Fortbildungen in Qualitätszirkeln, Supervisionen, aber auch Kongresse und Kurse besuchen. Für spezialisierte und qualifizierte Fachärzte, auch aus den Reihen der Allgemeinen und Inneren Medizin, erhoffe ich mir, dass das Bundesamt für Gesundheit BAG die Diagnosestellung und konsekutive Stimulanzientherapie zulasten der Grundversicherung zulässt, d.h. bei den Stimulanzien (vorerst lediglich bei Concerta ${ }^{\circledast}$ und Focalin $\mathrm{XR}^{\oplus}$ ) die Limitation in der Spezialitätenliste leicht korrigiert. Ich bin überzeugt, dass es so zu einer qualitativ besseren Versorgung der ADHS-Patienten kommt. Für Fachärzte der Kern-Fachgesellschaften müsste der ADHS-spezifische Fortbildungsaufwand in bescheidenem Rahmen ausfallen. Fachärzte anderer Spezialitäten hätten aber einige Fortbildungen inkl. psychiatrische zu absolvieren. Wesentlich ist, wie es bei jeglicher ärztlichen Tätigkeit gelten sollte, dass das Kennen seiner fachlichen Grenzen und das sich in Eigenverantwortung daran Halten wesentlich zur Qualität beitragen. Keinesfalls möchte ich einer Überreglementierung von Swissmedic und BAG Vorschub leisten, sondern durch ärztlicherseits formulierte Qualitätskriterien zu rigiden Vorschriften zuvorkommen.

1 Methylphenidat ist ein Arzneistoff aus der Gruppe der Stimulanzien. Er gehört zu den Amphetamin-ähnlichen Substanzen, die auch bei einer Aufmerksamkeitsdefizit-/ Hyperaktivitätsstörung (ADHS) verabreicht werden. Ritalin ${ }^{\oplus}$ ist das bekannteste Methylphenidat-Präparat.

2 Die Aufmerksamkeitsdefizit-/Hyperaktivitätsstörung (ADHS) ist eine bereits im Kindesalter beginnende neuropsychiatrische Störung, die sich durch Probleme mit der Aufmerksamkeit sowie Impulsivität und oft auch Hyperaktivität auszeichnet. Der unaufmerksame Subtyp (ohne Hyperaktivität) kommt öfters bei Mädchen und Erwachsenen vor. 